\title{
Parameter Estimation of the Weighted Generalized Inverse Weibull Distribution
}

\author{
Sofi Mudasir ${ }^{*}$, S.P. Ahmad \\ Department of Statistics, University of Kashmir, Srinagar 190 006, India
}

\section{ARTICLE INFO}

Article History

Received 02 Aug 2018

Accepted 28 Nov 2020

Keywords

Generalized inverse Weibull distribution

Weighted generalized inverse

Weibull distribution

Loss function

Bayesian estimation.

2000 Mathematics Subject

Classification:22E46,53C $35,57 \mathrm{~S} 20$

\begin{abstract}
Weighted distributions are used widely in many fields of real life such as medicine, ecology, reliability, and so on. The idea of weighted distributions was given by Fisher and studied by Rao in a unified manner who pointed out that in many situations the recorded observations cannot be considered as a random sample from the original distribution. This can be due to nonobservability of some events, damage caused to the original observations or adoption of unequal probability sampling procedure. In this paper, we have proposed weighted version of generalized inverse Weibull distribution known as weighted generalized inverse Weibull distribution (WGIWD). Classical and Bayesian methods of estimation were proposed for estimating the parameters of the new model. The usefulness of the new model was demonstrated by applying it to a real-life data set.
\end{abstract}

(c) 2021 The Authors. Published by Atlantis Press B.V. This is an open access article distributed under the CC BY-NC 4.0 license (http://creativecommons.org/licenses/by-nc/4.0/).

\section{INTRODUCTION}

In many observational studies for wild life, human, fish population or insect, every unit in the population does not have the same chance of being included in the sample. In such cases, sampling frames are not well defined and recorded observations are biased. These observations don't follow the parent distribution and hence their modeling gives birth to the theory of weighted distributions. Fisher [1] and Rao [2] introduced and unified the concept of weighted distribution. Rao identified various situations that can be modeled by weighted distributions.

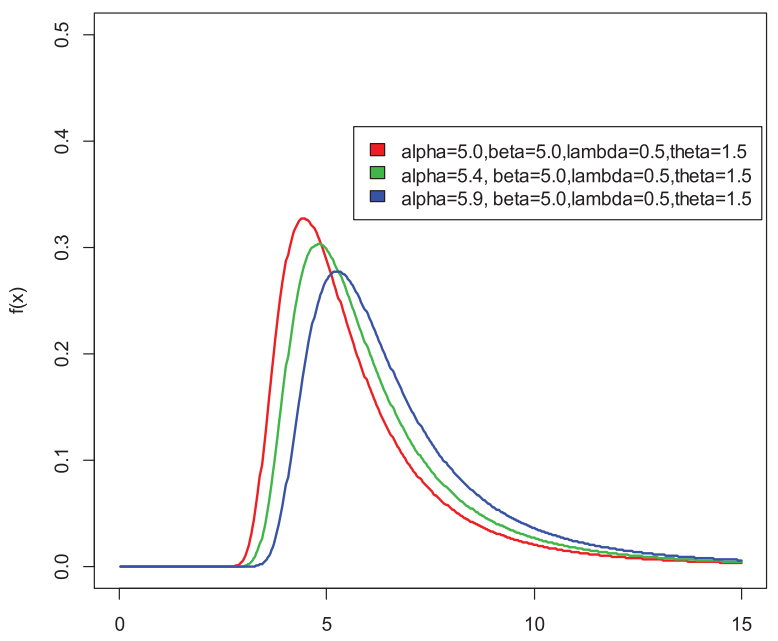

Figure 1 Probability distribution function of weighted generalized inverse Weibull distribution. 


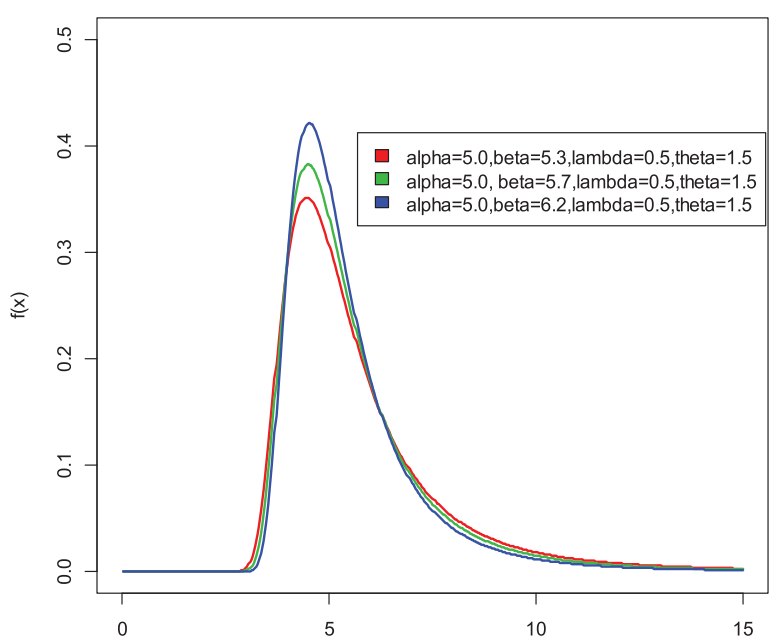

Figure 2 Probability distribution function of weighted generalized inverse Weibull distribution.

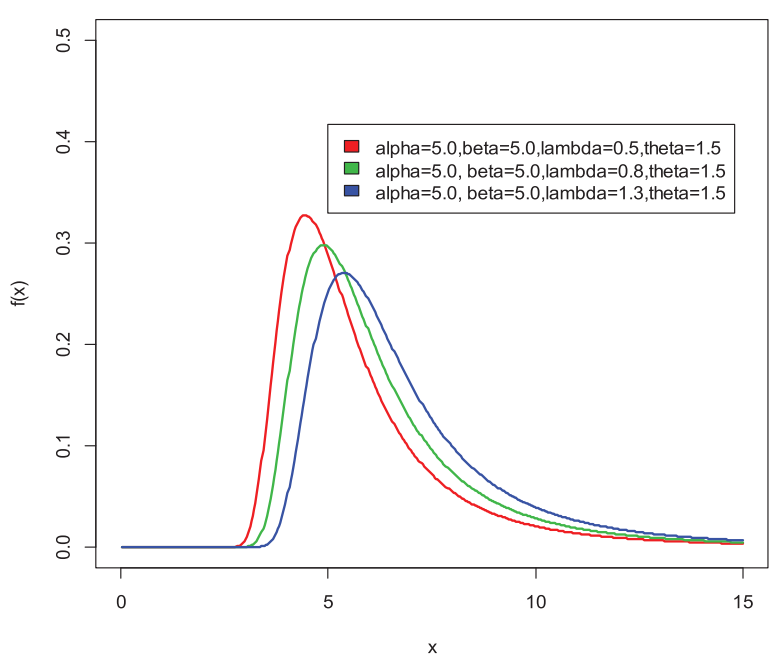

Figure 3 Probability distribution function of weighted generalized inverse Weibull distribution.

These situations refer to instances where the recorded observations cannot be considered as a random sample from the original distributions. This may occur due to nonobservability of some events or damage caused to the original observation, or adoption of unequal probability sampling procedure. Weighted distributions were used frequently in research related to reliability, biomedicine, ecology and branching processes can be seen in Patil and Rao [3], Gupta and Kirmani [4], Gupta and Keating [5], Oluyede [6] and in references there in. There are many researchers for weighted distribution as Das and Roy [7] discussed the length-biased weighted generalized Rayleigh distribution with its properties, Sofi et al. [8] studied the structural properties of length-biased Nakagami distribution. For more important results of weighted distribution see Oluyede and George [9], Ghitany and Al-Mutairi [10], Ahmed, Reshi and Mir [11], Sofi et al. [12].

Suppose $\mathrm{X}$ is a nonnegative random variable with probability density function $f(x)$, then the probability density function of the weighted random variable is given by

$$
f_{w}(x)=\frac{w(x) f(x)}{\mu_{w}}, x>0,
$$

where $w(x)$ be a nonnegative weight function and $\mu_{w}=E(w(x))<\infty$

The probability density function of generalized inverse Weibull distribution is given by

$$
f(x)=\frac{\lambda \beta \alpha^{\beta}}{x^{\beta+1}} \exp \left(-\lambda\left(\frac{\alpha}{x}\right)^{\beta}\right) \quad x>0 ; \alpha, \beta, \lambda>0
$$




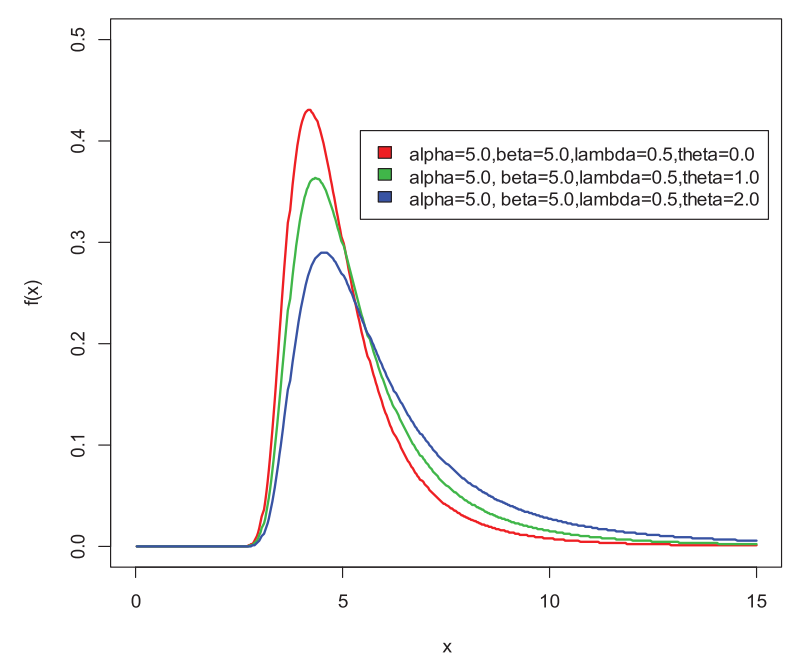

Figure 4 Probability distribution function of weighted generalized inverse Weibull distribution.

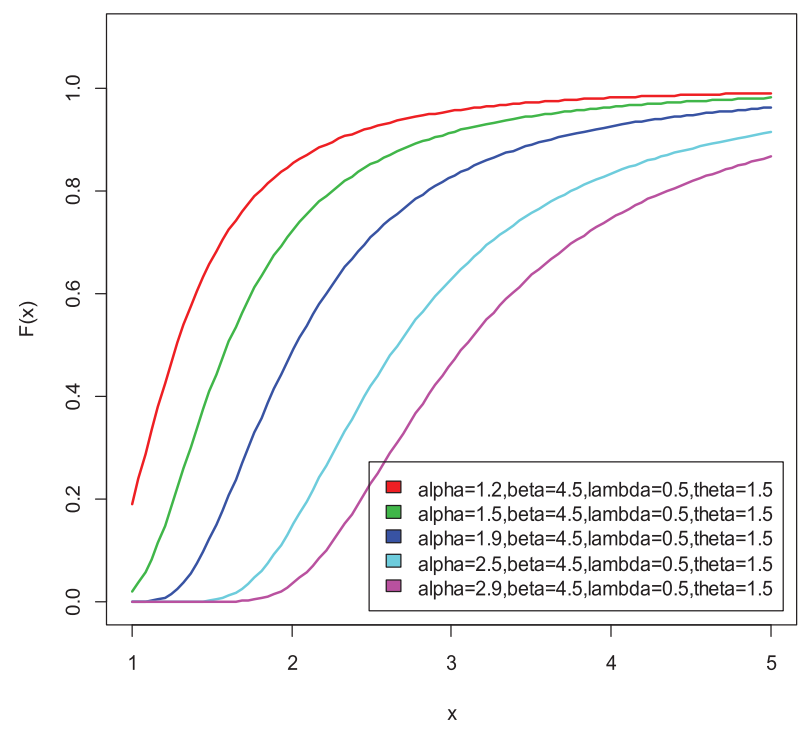

Figure 5 Cumulative distribution function of weighted generalized inverse Weibull distribution.

where $\alpha$ and $\beta$ are called scale and shape parameters, respectively.

Let

$$
w(x)=x^{\theta}, \theta>0 \leq
$$

Now, $\mu_{w}=\int_{0}^{\infty} w(x) f(x) d x$

$$
\Rightarrow \quad \mu_{w}=\alpha^{\theta} \lambda^{\frac{\theta}{\beta}} \Gamma\left(1-\frac{\theta}{\beta}\right), \quad \frac{\theta}{\beta}<1
$$




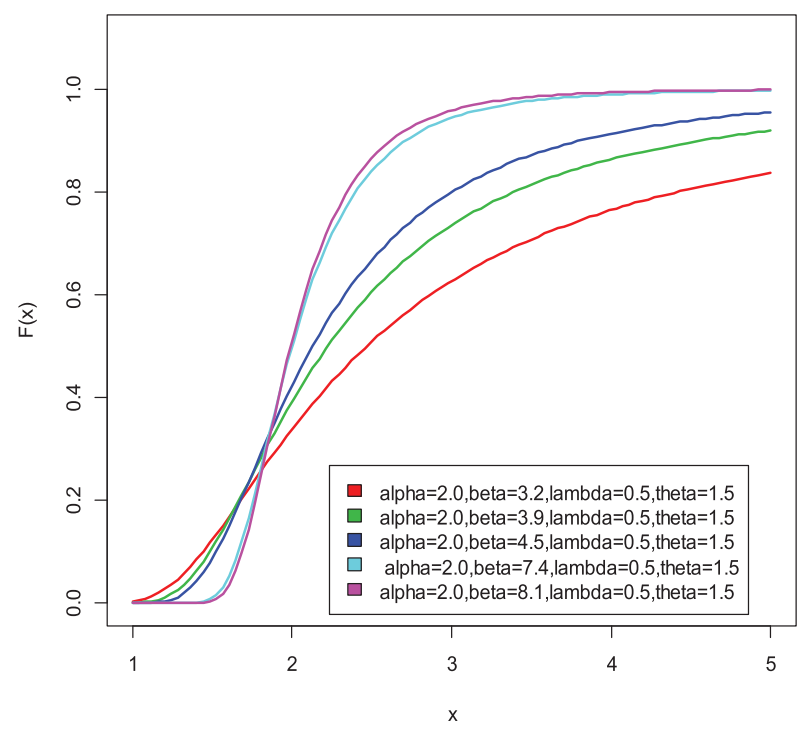

Figure 6 Cumulative distribution function of weighted generalized inverse Weibull distribution.

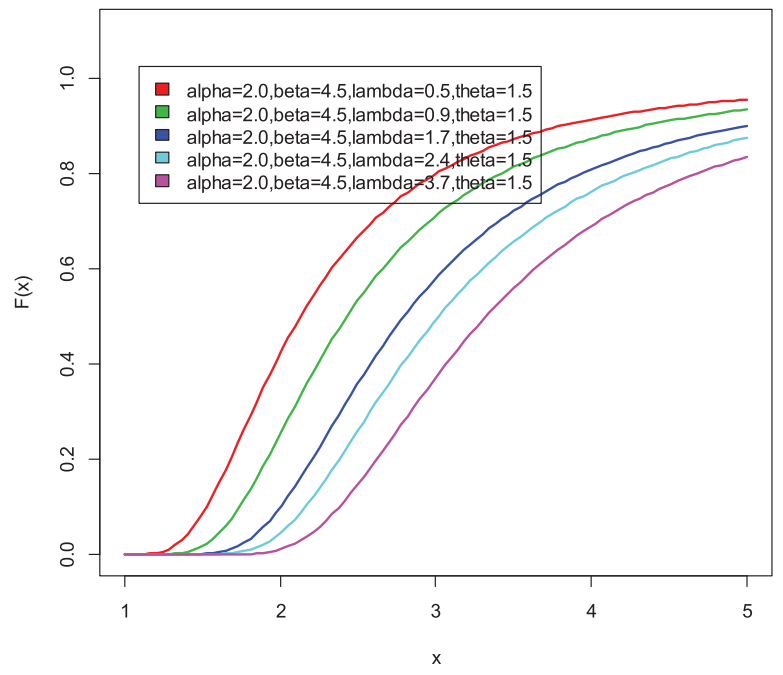

Figure 7 Cumulative distribution function of weighted generalized inverse Weibull distribution.

Substitute the value of Equations (2)-(4) in Equation (1), we get

$$
f_{w}(x)=\frac{\beta \alpha^{\beta-\theta} \lambda^{1-\frac{\theta}{\beta}} x^{\theta-\beta-1} \exp \left(-\lambda\left(\frac{\alpha}{x}\right)^{\beta}\right)}{\Gamma\left(1-\frac{\theta}{\beta}\right)}, x>0 ; \alpha, \beta, \theta, \lambda>0 \text { and } \theta<\beta
$$

The density function in Equation (5) is known as weighted generalized inverse Weibull distribution (WGIWD).

Also the cumulative distribution function (cdf) of weighted generalized inverse Weibull distribution (WGIWD) is

$$
F_{w}(x)=\frac{\Gamma\left(1-\frac{\theta}{\beta}, \frac{\lambda \alpha^{\beta}}{x^{\beta}}\right)}{\Gamma\left(1-\frac{\theta}{\beta}\right)}
$$




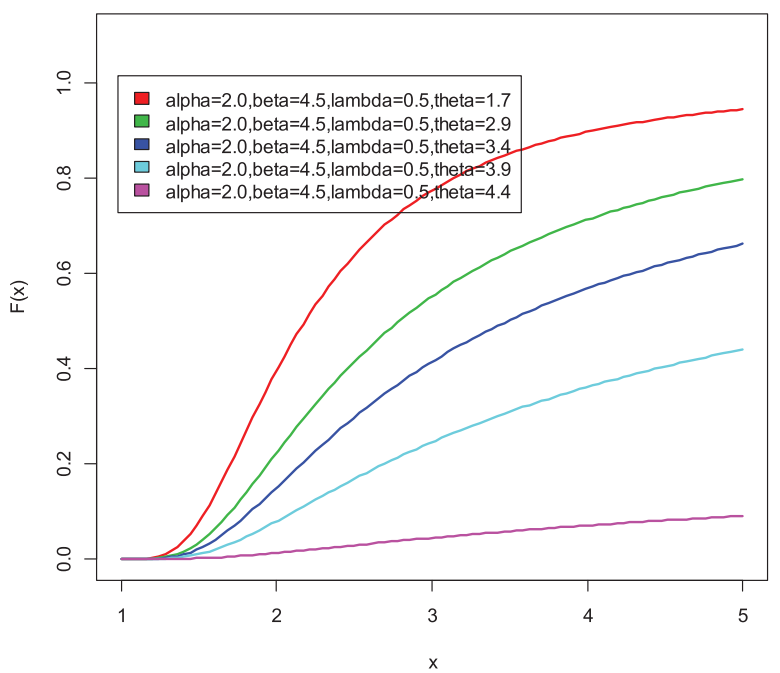

Figure 8 Cumulative distribution function of weighted generalized inverse Weibull distribution.

\section{MAXIMUM LIKELIHOOD ESTIMATION}

Let $x_{1}, x_{2}, \ldots, x_{n}$ be a random sample from (5), then the likelihood function is given by

$$
\begin{gathered}
L=\left(\frac{\beta \alpha^{\beta-\theta} \lambda^{1-\frac{\theta}{\beta}}}{\Gamma\left(1-\frac{\theta}{\beta}\right)}\right)^{n} \prod_{i=1}^{n} x_{i}^{\theta-\beta-1} \exp \left(-\lambda \alpha^{\beta} \sum_{i=1}^{n} x_{i}^{-\beta}\right) \\
\Rightarrow \quad \log L=n \log \beta+n(\beta-\theta) \log \alpha+n\left(1-\frac{\theta}{\beta}\right) \log \lambda-n \log \Gamma\left(1-\frac{\theta}{\beta}\right)+(\theta-\beta-1) \sum_{i=1}^{n} \log x_{i}-\lambda \alpha^{\beta} \sum_{i=1}^{n} x_{i}^{-\beta}
\end{gathered}
$$

Now differentiate the above equation with respect to $\alpha$ and equate to zero, we get

$$
\hat{\alpha}=\left(\frac{n(\beta-\theta)}{\lambda \beta \sum_{i=1}^{n} x_{i}^{-\beta}}\right)^{\frac{1}{\beta}}
$$

This is the required MLE of $\alpha$

\section{PARAMETER ESTIMATION UNDER SQUARED ERROR LOSS FUNCTION}

In this section two different prior distributions namely Jeffrey's prior and extension of Jeffrey's prior are used for estimating the scale parameter of the WGIWD.

\subsection{Using Jeffrey's Prior}

Consider that the parameter $\alpha$ has Jeffrey's prior given by

$$
\pi_{1}(\alpha) \propto \sqrt{\operatorname{det}(I(\alpha))}, \alpha>0
$$


where $I(\alpha)$ is the fisher information matrix obtained as

$$
\begin{aligned}
I(\alpha) & =-n E\left(\frac{\partial^{2} \log \left(f_{w}(x)\right)}{\partial \alpha^{2}}\right) \\
& =\frac{n \beta(\beta-\theta)}{\alpha^{2}}
\end{aligned}
$$

Therefore, the Jeffrey's prior distribution is defined by

$$
\pi_{1}(\alpha) \propto \frac{1}{\alpha}
$$

The posterior distribution using Jeffrey's prior is obtained by using Bayes theorem given by

$$
\begin{gathered}
P_{1}(\alpha \mid x) \propto L(\alpha \mid x) \pi_{1}(\alpha) \\
\Rightarrow \quad P_{1}(\alpha \mid x)=k\left(\frac{\beta \lambda^{1-\frac{\theta}{\beta}}}{\Gamma\left(1-\frac{\theta}{\beta}\right)}\right)^{n} \eta^{\theta-\beta-1} \alpha^{n(\beta-\theta)-1} \exp \left(-\lambda \alpha^{\beta} T\right)
\end{gathered}
$$

where $\eta=\prod_{i=1}^{n} x_{i}, \quad T=\sum_{i=1}^{n} x_{i}^{-\beta}$ and $k$ is the normalizing constant and is given by

$$
k=\frac{\Gamma^{n}\left(1-\frac{\theta}{\beta}\right) T^{n\left(1-\frac{\theta}{\beta}\right)}}{\Gamma\left(n\left(1-\frac{\theta}{\beta}\right)\right) \beta^{n-1} \eta^{\theta-\beta-1}}
$$

With this value of $k$ we get from (7) the posterior distribution as

$$
P_{1}(\alpha \mid x)=\frac{\beta(\lambda T)^{n\left(1-\frac{\theta}{\beta}\right)}}{\Gamma\left(n\left(1-\frac{\theta}{\beta}\right)\right)} \alpha^{n(\beta-\theta)-1} \exp \left(-\lambda \alpha^{\beta} T\right)
$$

By using squared error loss function $L(\hat{\alpha}, \alpha)=c(\hat{\alpha}-\alpha)^{2}$, for some constant $c$, the risk function is given by

$$
R(\hat{\alpha})=c \hat{\alpha}^{2}+c \frac{\Gamma\left(\frac{n(\beta-\theta)+2}{\beta}\right)}{\Gamma\left(n\left(1-\frac{\theta}{\beta}\right)\right)(\lambda T)^{\frac{2}{\beta}}}-2 c \hat{\alpha} \frac{\Gamma\left(\frac{n(\beta-\theta)+1}{\beta}\right)}{\Gamma\left(n\left(1-\frac{\theta}{\beta}\right)\right)(\lambda T)^{\frac{1}{\beta}}}
$$

The Bayes estimator $\hat{\alpha}$ is the solution of the equation $\frac{\partial R(\hat{\alpha})}{\partial \hat{\alpha}}=0$, which results in

$$
\hat{\alpha}=\frac{\Gamma\left(\frac{n(\beta-\theta)+1}{\beta}\right)}{\Gamma\left(\frac{n(\beta-\theta)}{\beta}\right)(\lambda T)^{\frac{1}{\beta}}}
$$

\subsection{Using Extension of Jeffrey's Prior}

The extension of Jeffrey's prior relating to the scale parameter $\alpha$ is given as

$$
\pi_{2}(\alpha) \propto(\sqrt{\operatorname{det}(I(\alpha))})^{C_{1}}, C_{1} \in R^{+}
$$

where $I(\alpha)$ is same as in Jeffrey's prior.

$$
\Rightarrow \quad \pi_{2}(\alpha) \propto \frac{1}{\alpha^{2 C_{1}}}
$$


The posterior distribution using extension of Jeffrey's prior is obtained by using the same procedure as in case of Jeffrey's prior and is given by

$$
P_{2}(\alpha \mid x)=\frac{\beta(\lambda T)^{\frac{n(\beta-\theta)-2 C_{1}+1}{\beta}}}{\Gamma\left(\frac{n(\beta-\theta)-2 C_{1}+1}{\beta}\right)} \alpha^{n(\beta-\theta)-2 C_{1}} \exp \left(-\lambda \alpha^{\beta} T\right)
$$

By using squared error loss function $L(\hat{\alpha}, \alpha)=c(\hat{\alpha}-\alpha)^{2}$, for some constant $c$, the risk function is given by

$$
R(\hat{\alpha})=c \hat{\alpha}^{2}+c \frac{\Gamma\left(\frac{n(\beta-\theta)-2 C_{1}+3}{\beta}\right)}{\Gamma\left(\frac{n(\beta-\theta)-2 C_{1}+1}{\beta}\right)(\lambda T)^{\frac{2}{\beta}}}-2 c \hat{\alpha} \frac{\Gamma\left(\frac{n(\beta-\theta)-2 C_{1}+2}{\beta}\right)}{\Gamma\left(\frac{n(\beta-\theta)-2 C_{1}+1}{\beta}\right)(\lambda T)^{\frac{1}{\beta}}}
$$

The Bayes estimator $\hat{\alpha}$ is the solution of the equation $\frac{\partial R(\hat{\alpha})}{\partial \hat{\alpha}}=0$, which results in

$$
\hat{\alpha}=\frac{\Gamma\left(\frac{n(\beta-\theta)-2 C_{1}+2}{\beta}\right)}{(\lambda T)^{\frac{1}{\beta}} \Gamma\left(\frac{n(\beta-\theta)-2 C_{1}+1}{\beta}\right)}
$$

Remark 1. If $C_{1}=\frac{1}{2}$ in (11), the same Bayes estimator is obtained as in (9) corresponding to Jeffrey's prior.

\section{PARAMETER ESTIMATION UNDER QUADRATIC LOSS FUNCTION}

In this section we use quadratic loss function to obtain Bayes estimators using Jeffrey's and extension of Jeffrey's prior information. The quadratic loss function is defined as

$$
L(\hat{\alpha}, \alpha)=\left(\frac{\alpha-\hat{\alpha}}{\alpha}\right)^{2}
$$

\subsection{Using Jeffrey's Prior}

By using the quadratic loss function $L(\hat{\alpha}, \alpha)=\left(\frac{\alpha-\hat{\alpha}}{\alpha}\right)^{2}$, the risk function is given by

$$
R(\hat{\alpha})=1+\hat{\alpha}^{2}(\lambda T)^{\frac{2}{\beta}} \frac{\Gamma\left(\frac{n(\beta-\theta)-2}{\beta}\right)}{\Gamma\left(n\left(1-\frac{\theta}{\beta}\right)\right)}-2 \hat{\alpha}(\lambda T)^{\frac{1}{\beta}} \frac{\Gamma\left(\frac{n(\beta-\theta)-1}{\beta}\right)}{\Gamma\left(n\left(1-\frac{\theta}{\beta}\right)\right)}
$$

The Bayes estimator $\hat{\alpha}$ is the solution of the equation $\frac{\partial R(\hat{\alpha})}{\partial \hat{\alpha}}=0$, which results in

$$
\hat{\alpha}=\frac{\Gamma\left(\frac{n(\beta-\theta)-1}{\beta}\right)}{(\lambda T)^{\frac{1}{\beta}} \Gamma\left(\frac{n(\beta-\theta)-2}{\beta}\right)}
$$

\subsection{Using Extension of Jeffrey's Prior}

Taking the posterior distribution (11) and by using the quadratic loss function, the risk function is given by

$$
R(\hat{\alpha})=1+\hat{\alpha}^{2}(\lambda T)^{\frac{2}{\beta}} \frac{\Gamma\left(\frac{n(\beta-\theta)-2 C_{1}-1}{\beta}\right)}{\Gamma\left(\frac{n(\beta-\theta)-2 C_{1}+1}{\beta}\right)}-2 \hat{\alpha}(\lambda T)^{\frac{1}{\beta}} \frac{\Gamma\left(\frac{n(\beta-\theta)-2 C_{1}}{\beta}\right)}{\Gamma\left(\frac{n(\beta-\theta)-2 C_{1}+1}{\beta}\right)}
$$


The Bayes estimator $\hat{\alpha}$ is the solution of the equation $\frac{\partial R(\hat{\alpha})}{\partial \hat{\alpha}}=0$, which results in

$$
\hat{\alpha}=\frac{\Gamma\left(\frac{n(\beta-\theta)-2 C_{1}}{\beta}\right)}{(\lambda T)^{\frac{1}{\beta}} \Gamma\left(\frac{n(\beta-\theta)-2 C_{1}-1}{\beta}\right)}
$$

Remark 2. Replacing $C_{1}=\frac{1}{2}$ in (13), the same Bayes estimator is obtained as in (12) corresponding to Jeffrey's prior.

\section{PARAMETER ESTIMATION UNDER NEW LOSS FUNCTION}

In this section we obtain the Bayes estimators under new loss function introduced by Al-Bayyati [13] using Jeffrey's and extension of Jeffrey's prior

The Al-Bayyati's new loss function also called new loss function is of the form

$$
L(\hat{\alpha}, \alpha)=\alpha^{C_{2}}(\hat{\alpha}-\alpha)^{2}, C_{2} \in R
$$

Here we use this loss function to obtain the Bayes estimator of the scale parameter $\alpha$ of the WGIWD.

\subsection{Using Jeffrey's Prior}

By using the new loss function $L(\hat{\alpha}, \alpha)=\alpha^{C_{2}}(\hat{\alpha}-\alpha)^{2}$, the risk function is given by

$$
R(\hat{\alpha})=\hat{\alpha}^{2} \frac{\Gamma\left(\frac{n(\beta-\theta)+C_{2}}{\beta}\right)}{(\lambda T)^{\frac{C_{2}}{\beta}} \Gamma\left(n\left(1-\frac{\theta}{\beta}\right)\right)}+\frac{\Gamma\left(\frac{n(\beta-\theta)+C_{2}+2}{\beta}\right)}{(\lambda T)^{\frac{C_{2}+2}{\beta}} \Gamma\left(n\left(1-\frac{\theta}{\beta}\right)\right)}-2 \hat{\alpha} \frac{\Gamma\left(\frac{n(\beta-\theta)+C_{2}+1}{\beta}\right)}{(\lambda T)^{\frac{C_{2}+1}{\beta}} \Gamma\left(n\left(1-\frac{\theta}{\beta}\right)\right)}
$$

The Bayes estimator $\hat{\alpha}$ is the solution of the equation $\frac{\partial R(\hat{\alpha})}{\partial \hat{\alpha}}=0$, which results in

$$
\hat{\alpha}=\frac{\Gamma\left(\frac{n(\beta-\theta)+C_{2}+1}{\beta}\right)}{(\lambda T)^{\frac{1}{\beta}} \Gamma\left(\frac{n(\beta-\theta)+C_{2}}{\beta}\right)}
$$

Remark 3. The Bayes estimator obtained under Jeffrey's prior given in (14) coincides with the Bayes estimator given in (9) if $C_{2}=0$ in (14).

\subsection{Using Extension of Jeffrey's Prior}

By taking the posterior distribution (11) and using the new loss function, the risk function is given by

$$
R(\hat{\alpha})=\hat{\alpha}^{2} \frac{\Gamma\left(\frac{n(\beta-\theta)-2 C_{1}+C_{2}+1}{\beta}\right)}{(\lambda T)^{\frac{C_{2}}{\beta}} \Gamma\left(\frac{n(\beta-\theta)-2 C_{1}+1}{\beta}\right)}+\frac{\Gamma\left(\frac{n(\beta-\theta)-2 C_{1}+C_{2}+3}{\beta}\right)}{(\lambda T)^{\frac{C_{2}+2}{\beta}} \Gamma\left(\frac{n(\beta-\theta)-2 C_{1}+1}{\beta}\right)}-2 \hat{\alpha} \frac{\Gamma\left(\frac{n(\beta-\theta)-2 C_{1}+C_{2}+2}{\beta}\right)}{(\lambda T)^{\frac{C_{2}+1}{\beta}} \Gamma\left(\frac{n(\beta-\theta)-2 C_{1}+1}{\beta}\right)}
$$

The Bayes estimator $\hat{\alpha}$ is the solution of the equation $\frac{\partial R(\hat{\alpha})}{\partial \hat{\alpha}}=0$, which results in

$$
\hat{\alpha}=\frac{\Gamma\left(\frac{n(\beta-\theta)-2 C_{1}+C_{2}+2}{\beta}\right)}{(\lambda T)^{\frac{1}{\beta}} \Gamma\left(\frac{n(\beta-\theta)-2 C_{1}+C_{2}+1}{\beta}\right)}
$$

Remark 4. If $C_{1}=\frac{1}{2}$ in (15), the same Bayes estimator is obtained as in (14) corresponding to Jeffrey's prior and if $C_{2}=0$ in (15) the Bayes estimator coincides with the Bayes estimator given in (11). 


\section{POSTERIOR MEAN AND POSTERIOR VARIANCE OF SCALE PARAMETER UNDER JEFFREY'S AND EXTENSION OF JEFFREY'S PRIORS}

In this section, we calculate the posterior mean and posterior variance of the scale parameter $\alpha$ under Jeffrey's and extension of Jeffrey's Prior distribution.

\subsection{Posterior Mean and Posterior Variance of Under Jeffrey's Prior}

We have the posterior distribution under Jeffrey's prior as

$$
P_{1}(\alpha \mid x)=\frac{\beta(\lambda T)^{n\left(1-\frac{\theta}{\beta}\right)}}{\Gamma\left(n\left(1-\frac{\theta}{\beta}\right)\right)} \alpha^{n(\beta-\theta)-1} \exp \left(-\lambda \alpha^{\beta} T\right)
$$

Now

$$
E\left(\alpha^{r}\right)=\int_{0}^{\infty} \alpha^{r} P_{1}(\alpha \mid x) d \alpha
$$

By using Equation (16) in Equation (17), we get

$$
E\left(\alpha^{r}\right)=\frac{\Gamma\left(\frac{n(\beta-\theta)+r}{\beta}\right)}{(\lambda T)^{\frac{r}{\beta}} \Gamma\left(\frac{n(\beta-\theta)}{\beta}\right)}
$$

If $r=1$ in (18), we get

$$
E(\alpha)=\frac{\Gamma\left(\frac{n(\beta-\theta)+1}{\beta}\right)}{(\lambda T)^{\frac{1}{\beta}} \Gamma\left(\frac{n(\beta-\theta)}{\beta}\right)}
$$

This is the posterior mean

If $r=2$ in (18), we get

$$
E\left(\alpha^{2}\right)=\frac{\Gamma\left(\frac{n(\beta-\theta)+2}{\beta}\right)}{(\lambda T)^{\frac{2}{\beta}} \Gamma\left(\frac{n(\beta-\theta)}{\beta}\right)}
$$

Thus the posterior variance is given by

$$
v(\alpha)=\frac{\Gamma\left(\frac{n(\beta-\theta)}{\beta}\right) \Gamma\left(\frac{n(\beta-\theta)+2}{\beta}\right)-\Gamma^{2}\left(\frac{n(\beta-\theta)+1}{\beta}\right)}{(\lambda T)^{\frac{2}{\beta}} \Gamma^{2}\left(\frac{n(\beta-\theta)}{\beta}\right)}
$$

\subsection{Posterior Mean and Posterior Variance of Under Extension of Jeffrey's Prior}

We have the posterior distribution under extension of Jeffrey's prior as

$$
P_{2}(\alpha \mid x)=\frac{\beta(\lambda T)^{\frac{n(\beta-\theta)-2 C_{1}+1}{\beta}}}{\Gamma\left(\frac{n(\beta-\theta)-2 C_{1}+1}{\beta}\right)} \alpha^{n(\beta-\theta)-2 C_{1}} \exp \left(-\lambda \alpha^{\beta} T\right)
$$


Now

$$
E\left(\alpha^{r}\right)=\int_{0}^{\infty} \alpha^{r} P_{2}(\alpha \mid x) d \alpha
$$

By using Equation (19) in Equation (20), we get

$$
E\left(\alpha^{r}\right)=\frac{\Gamma\left(\frac{n(\beta-\theta)-2 C_{1}+r+1}{\beta}\right)}{(\lambda T)^{\frac{r}{\beta}} \Gamma\left(\frac{n(\beta-\theta)-2 C_{1}+1}{\beta}\right)}
$$

If $r=1$ in (21), we get

$$
E(\alpha)=\frac{\Gamma\left(\frac{n(\beta-\theta)-2 C_{1}+2}{\beta}\right)}{(\lambda T)^{\frac{1}{\beta}} \Gamma\left(\frac{n(\beta-\theta)-2 C_{1}+1}{\beta}\right)}
$$

This is the posterior mean

If $r=2$ in (21), we get

$$
E\left(\alpha^{2}\right)=\frac{\Gamma\left(\frac{n(\beta-\theta)-2 C_{1}+3}{\beta}\right)}{(\lambda T)^{\frac{2}{\beta}} \Gamma\left(\frac{n(\beta-\theta)-2 C_{1}+1}{\beta}\right)}
$$

Thus the posterior variance is given by

$$
\Rightarrow \quad v(\alpha)=\frac{\Gamma\left(\frac{n(\beta-\theta)-2 C_{1}+1}{\beta}\right) \Gamma\left(\frac{n(\beta-\theta)-2 C_{1}+3}{\beta}\right)-\Gamma^{2}\left(\frac{n(\beta-\theta)-2 C_{1}+2}{\beta}\right)}{(\lambda T)^{\frac{2}{\beta}} \Gamma^{2}\left(\frac{n(\beta-\theta)-2 C_{1}+1}{\beta}\right)}
$$

Remark 5. If $C_{1}=\frac{1}{2}$ then the posterior mean and posterior variance obtained under extension of Jeffrey's prior coincides with the posterior mean and posterior variance obtained under Jeffrey's prior.

\section{DATA ANALYSIS}

In this section we analyze real-life data set for illustration given by Lee and Wang [14] which represent remission times (in months) of a random sample of 128 bladder cancer patients (Table 1). A program has been developed in R language to obtain the Bayes estimates and posterior risks. The data are as follows: $0.08,2.09,3.48,4.87,6.94,8.66,13.11,23.63,0.20,2.23,3.52,4.98,6.97,9.02,13.29,0.40,2.26,3.57$, $5.06,7.09,9.22,13.80,25.74,0.50,2.46,3.64,5.09,7.26,9.47,14.24,25.82,0.51,2.54,3.70,5.17,7.28,9.74,14.76,26.31,0.81,2.62,3.82,5.32$, $7.32,10.06,14.77,32.15,2.64,3.88,5.32,7.39,10.34,14.83,34.26,0.90,2.69,4.18,5.34,7.59,10.66,15.96,36.66,1.05,2.69,4.23,5.41,7.62$, $10.75,16.62,43.01,1.19,2.75,4.26,5.41,7.63,17.12,46.12,1.26,2.83,4.33,5.49,7.66,11.25,17.14,79.05,1.35,2.87,5.62,7.87,11.64,17.36$, $1.40,3.02,4.34,5.71,7.93,11.79,18.10,1.46,4.40,5.85,8.26,11.98,19.13,1.76,3.25,4.50,6.25,8.37,12.02,2.02,3.31,4.51,6.54,8.53,12.03$, $20.28,2.02,3.36,6.76,12.07,21.73,2.07,3.36,6.93,8.65,12.63,22.69$.

By using different loss functions, that is, SELF, QLF and NLF, the Bayes estimates and posterior risk through Jeffrey's and extension of Jeffrey's priors are presented in the tables below where posterior risk are in parentheses.

Table 1 Descriptive statistics for the above real data set.

\begin{tabular}{lcccccccc}
\hline Min. & 1st Qu. & Median & Mean & 3rd Qu. & Max. & $\begin{array}{l}\text { Standard } \\
\text { deviation }\end{array}$ & Skewness & Kurtosis \\
\hline 0.080 & 3.348 & 6.395 & 9.366 & 11.840 & 79.050 & 10.50833 & 3.286569 & 18.48308 \\
\hline
\end{tabular}


Table 2 Estimates and (posterior risk) of $\alpha$ under Jeffrey's prior.

\begin{tabular}{|c|c|c|c|c|c|c|c|c|}
\hline$\lambda$ & $\beta$ & $\theta$ & C & $C_{2}$ & MLE & SELF & QLF & NLF \\
\hline \multirow{4}{*}{1.05} & 1.5 & 0.5 & 1 & 0.5 & 0.7981785 & $\begin{array}{c}0.7971406 \\
(0.003311692)\end{array}$ & $\begin{array}{c}0.7887990 \\
(0.005266604)\end{array}$ & $\begin{array}{c}0.7992192 \\
(0.0029587057)\end{array}$ \\
\hline & 2.0 & 0.5 & 3 & 1.0 & 0.8956767 & $\begin{array}{c}0.8945112 \\
(0.006259293)\end{array}$ & $\begin{array}{c}0.8898279 \\
(0.002628107)\end{array}$ & $\begin{array}{c}0.8968437 \\
(0.0018663487)\end{array}$ \\
\hline & 2.5 & 1.0 & 4 & 1.5 & 0.8374464 & $\begin{array}{c}0.8361384 \\
(0.005839690)\end{array}$ & $\begin{array}{c}0.8326298 \\
(0.002105796)\end{array}$ & $\begin{array}{c}0.8387554 \\
(0.0011153567)\end{array}$ \\
\hline & 3.5 & 1.5 & 6 & 2.0 & 0.8687908 & $\begin{array}{c}0.8675784 \\
(0.005057925)\end{array}$ & $\begin{array}{c}0.8656269 \\
(0.001127539)\end{array}$ & $\begin{array}{c}0.8695190 \\
(0.0006331024)\end{array}$ \\
\hline \multirow{4}{*}{3.97} & 1.5 & 0.5 & 1 & 0.5 & 0.3288763 & $\begin{array}{c}0.3284486 \\
(0.0005622312)\end{array}$ & $\begin{array}{c}0.3250116 \\
(0.005266605)\end{array}$ & $\begin{array}{c}0.3293051 \\
(0.0003224283)\end{array}$ \\
\hline & 2.0 & 0.5 & 3 & 1.0 & 0.4606284 & $\begin{array}{c}0.4600290 \\
(0.0016554805)\end{array}$ & $\begin{array}{c}0.4576205 \\
(0.002628793)\end{array}$ & $\begin{array}{c}0.4612285 \\
(0.0002538581)\end{array}$ \\
\hline & 2.5 & 1.0 & 4 & 1.5 & 0.4919450 & $\begin{array}{c}0.4911766 \\
(0.0020151567)\end{array}$ & $\begin{array}{c}0.4891155 \\
(0.002105852)\end{array}$ & $\begin{array}{c}0.4927139 \\
(0.0001732894)\end{array}$ \\
\hline & 3.5 & 1.5 & 6 & 2.0 & 0.5941366 & $\begin{array}{c}0.5933075 \\
(0.0023654540)\end{array}$ & $\begin{array}{c}0.5919729 \\
(0.001127542)\end{array}$ & $\begin{array}{c}0.5946346 \\
(0.0001384708)\end{array}$ \\
\hline \multirow{4}{*}{4.12} & 1.5 & 0.5 & 1 & 0.5 & 0.3208446 & $\begin{array}{c}0.3204274 \\
(0.0005351054)\end{array}$ & $\begin{array}{c}0.3170743 \\
(0.005266658)\end{array}$ & $\begin{array}{c}0.3212629 \\
(0.0003031018)\end{array}$ \\
\hline & 2.0 & 0.5 & 3 & 1.0 & 0.4521654 & $\begin{array}{c}0.4515770 \\
(0.0015952082)\end{array}$ & $\begin{array}{c}0.4492128 \\
(0.002628331)\end{array}$ & $\begin{array}{c}0.4527545 \\
(0.0002401214)\end{array}$ \\
\hline & 2.5 & 1.0 & 4 & 1.5 & 0.4847009 & $\begin{array}{c}0.4839439 \\
(0.0019562459)\end{array}$ & $\begin{array}{c}0.4819132 \\
(0.002105828)\end{array}$ & $\begin{array}{c}0.4854585 \\
(0.0001645215)\end{array}$ \\
\hline & 3.5 & 1.5 & 6 & 2.0 & 0.5878742 & $\begin{array}{c}0.5870538 \\
(0.0023158514)\end{array}$ & $\begin{array}{c}0.5857333 \\
(0.001127543)\end{array}$ & $\begin{array}{c}0.5883669 \\
(0.0001327244)\end{array}$ \\
\hline
\end{tabular}

$\mathrm{MLE}=$ maximum likelihood estimator, $\mathrm{SELF}=$ square error loss function, $\mathrm{QLF}=$ quadratic loss function, NLF $=$ new loss function.

Table 3 Estimates and (posterior risk) of $\alpha$ under extension of Jeffrey's prior.

\begin{tabular}{|c|c|c|c|c|c|c|c|c|c|}
\hline$\lambda$ & $\beta$ & $\theta$ & $\mathrm{C}$ & $C_{1}$ & $C_{2}$ & MLE & SELF & QLF & NLF \\
\hline \multirow{5}{*}{1.05} & 1.5 & 0.5 & 1 & 0.5 & 0.5 & 0.7981785 & $\begin{array}{c}0.7971406 \\
(0.003311692)\end{array}$ & $\begin{array}{c}0.7887990 \\
(0.005266604)\end{array}$ & $\begin{array}{c}0.7992192 \\
(0.0029587057)\end{array}$ \\
\hline & 2.0 & 0.5 & 3 & 1.0 & 1.0 & 0.8956767 & $\begin{array}{c}0.8921727 \\
(0.006259250)\end{array}$ & $\begin{array}{c}0.8874770 \\
(0.002641994)\end{array}$ & $\begin{array}{c}0.8945112 \\
(0.0018614567)\end{array}$ \\
\hline & 2.5 & 1.0 & 4 & 2.0 & 1.5 & 0.8374464 & $\begin{array}{c}0.8308672 \\
(0.005858038)\end{array}$ & $\begin{array}{c}0.8273250 \\
(0.002139587)\end{array}$ & $\begin{array}{c}0.8335090 \\
(0.0011082842)\end{array}$ \\
\hline & 3.5 & 1.5 & 6 & 2.5 & 2.0 & 0.8687908 & $\begin{array}{c}0.8636644 \\
(0.005092232)\end{array}$ & $\begin{array}{c}0.8616906 \\
(0.001145624)\end{array}$ & $\begin{array}{c}0.8656269 \\
(0.0006316363)\end{array}$ \\
\hline & 1.5 & 0.5 & 1 & 0.5 & 0.5 & 0.3288763 & $\begin{array}{c}0.3284486 \\
(0.0005622312)\end{array}$ & $\begin{array}{c}0.3250116 \\
(0.005266605)\end{array}$ & $\begin{array}{c}0.3293051 \\
(0.0003224283)\end{array}$ \\
\hline \multirow[t]{2}{*}{3.97} & 2.0 & 0.5 & 3 & 1.0 & 1.0 & 0.4606284 & $\begin{array}{c}0.4588263 \\
(0.0016554692)\end{array}$ & $\begin{array}{c}0.4564114 \\
(0.002642805)\end{array}$ & $\begin{array}{c}0.4600290 \\
(0.0002531927)\end{array}$ \\
\hline & 2.5 & 1.0 & 4 & 2.0 & 1.5 & 0.4919450 & $\begin{array}{c}0.4880801 \\
(0.0020214881)\end{array}$ & $\begin{array}{c}0.4859993 \\
(0.002143806)\end{array}$ & $\begin{array}{c}0.4896320 \\
(0.0001721906)\end{array}$ \\
\hline \multirow{5}{*}{4.12} & 3.5 & 1.5 & 6 & 2.5 & 2.0 & 0.5941366 & $\begin{array}{c}0.5906308 \\
(0.0023814988)\end{array}$ & $\begin{array}{c}0.5892810 \\
(0.001145851)\end{array}$ & $\begin{array}{c}0.5919729 \\
(0.0001381502)\end{array}$ \\
\hline & 1.5 & 0.5 & 1 & 0.5 & 0.5 & 0.3208446 & $\begin{array}{c}0.3204274 \\
(0.0005351054)\end{array}$ & $\begin{array}{c}0.3170743 \\
(0.005266658)\end{array}$ & $\begin{array}{c}0.3212629 \\
(0.0003031018)\end{array}$ \\
\hline & 2.0 & 0.5 & 3 & 1.0 & 1.0 & 0.4521654 & $\begin{array}{c}0.4503964 \\
(0.0015951972)\end{array}$ & $\begin{array}{c}0.4480259 \\
(0.002641997)\end{array}$ & $\begin{array}{c}0.4515770 \\
(0.0002394920)\end{array}$ \\
\hline & 2.5 & 1.0 & 4 & 2.0 & 1.5 & 0.4847009 & $\begin{array}{c}0.4808930 \\
(0.0019623923)\end{array}$ & $\begin{array}{c}0.4788428 \\
(0.002139695)\end{array}$ & $\begin{array}{c}0.4824220 \\
(0.0001634783)\end{array}$ \\
\hline & 3.5 & 1.5 & 6 & 2.5 & 2.0 & 0.5878742 & $\begin{array}{c}0.5844053 \\
(0.0023315597)\end{array}$ & $\begin{array}{c}0.5830697 \\
(0.001145638)\end{array}$ & $\begin{array}{c}0.5857333 \\
(0.0001324170)\end{array}$ \\
\hline
\end{tabular}

$\mathrm{MLE}=$ maximum likelihood estimator, SELF $=$ square error loss function, $\mathrm{QLF}=$ quadratic loss function, NLF $=$ new loss function. 


\section{CONCLUSION}

In this paper, we have primarily estimate the scale parameter of the new model known as WGID under Jeffrey's and extension of Jeffrey's prior distributions assuming different loss functions. For comparison, we use the real-life data set and the results are shown in the tables above.

Tables 2 and 3 show the estimates and the posterior risk in parentheses of the scale parameter $\alpha$ for different values of the parametersunder the Jeffrey's and extension of Jeffrey's priors. From the tables it is clear that the estimates and posterior risks obtained under extension of Jeffrey's prior coincides with the estimates obtained under Jeffrey's prior when the value of hyper-parameter $C_{1}=\frac{1}{2}$. Also on comparing the posterior risks under different loss functions, it is observed that NLF has less value of posterior risk than other loss functions. Thus we conclude that in our case it is the NLF which is more preferable than other loss functions.

\section{CONFLICTS OF INTEREST}

The authors declare they have no conflicts of interest.

\section{AUTHORS' CONTRIBUTIONS}

Authors developed the new model and performed the analytical calculations. They also discussed the results and contributed to the final manuscript.

\section{ACKNOWLEDGMENTS}

The authors are very much thankful to the reviewers for their valuable inputs to bring this research paper to this form.

\section{REFERENCES}

1. R.A. Fisher, Ann. Eugen. 6 (1934), 13-25.

2. C.R. Rao, in G.P. Patil (Eds.), Pergamon Press and Statistical Publishing Society Calcutta, 1965, pp. 320-332. https://www.jstor. org/stable/25049375.

3. G.P. Patil, C.R. Rao, Biometrics. 34 (1978), 179-184.

4. R.C. Gupta, S.N.U.A. Kirmani, Commun. Statist. 19 (1990), 3147-3162.

5. R.C. Gupta, J.P. Keating, Scan. J. Statist. 13 (1985), 49-56. https://www.jstor.org/stable/4616010.

6. B.O. Oluyede, Probab. Eng. Inf. Sci. 13 (1999), 169-185.

7. K.K. Das, T.D. Roy, Adv. Appl.Sci. Res. 2 (2011), 320-327.

8. S. Mudasir, S.P. Ahmad, Int. J. Mod. Mat. Sci. 13 (2015), 217-227.

9. B.O. Oluyede, E.O. George, Math. Probl. Eng. 8 (2000), 1-13.

10. M.E. Ghitany, D.K. Al-Mutairi, Metron-Int. J. Stat. 79 (2007), 299-311.

11. A. Ahmed, J.A. Reshi, K.A. Mir, IOSR J. Math. 5 (2013), 55-61.

12. S. Mudasir, S.P. Ahmad, Int. J. Mod. Math. Sci. 14 (2016), 147-159.

13. H.N. Al-Bayyati, Comparing Methods of Estimating Weibull Failure Models Using Simulation, Ph.D. Thesis, College of Administration and Economics, Baghdad University, Iraq, 2002.

14. E.T. Lee, J.W. Wang, Statistical Methods for Survival Data Analysis, third ed., John Wiley and Sons, New York, USA, 2003. 